Article

\title{
Prospects for Searching Thermal Effects, Non-Newtonian Gravity and Axion-Like Particles: Cannex Test of the Quantum Vacuum
}

\author{
Galina L. Klimchitskaya ${ }^{1,2, *(\mathbb{D}}$, Vladimir M. Mostepanenko ${ }^{1,2,3}{ }^{(0)}$, René I. P. Sedmik ${ }^{4}$ \\ and Hartmut Abele ${ }^{4}$ \\ 1 Central Astronomical Observatory at Pulkovo of the Russian Academy of Sciences, \\ Saint Petersburg 196140, Russia; vmostepa@gmail.com \\ 2 Institute of Physics, Nanotechnology and Telecommunications, Peter the Great Saint Petersburg \\ Polytechnic University, Saint Petersburg 195251, Russia \\ 3 Kazan Federal University, Kazan 420008, Russia \\ 4 Technische Universität Wien, Atominstitut, Stadionallee 2, 1020 Vienna, Austria; \\ rene.sedmik@tuwien.ac.at (R.I.P.S.); hartmut.abele@tuwien.ac.at (H.A.) \\ * Correspondence: g.klimchitskaya@gmail.com
}

Received: 18 February 2019; Accepted: 18 March 2019; Published: 20 March 2019

\begin{abstract}
We consider the CANNEx (Casimir And Non-Newtonian force EXperiment) test of the quantum vacuum intended for measuring the gradient of the Casimir pressure between two flat parallel plates at large separations and constraining parameters of the chameleon model of dark energy in cosmology. A modification of the measurement scheme is proposed that allows simultaneous measurements of both the Casimir pressure and its gradient in one experiment. It is shown that with several improvements the CANNEX test will be capable to strengthen the constraints on the parameters of the Yukawa-type interaction by up to an order of magnitude over a wide interaction range. The constraints on the coupling constants between nucleons and axion-like particles, which are considered as the most probable constituents of dark matter, could also be strengthened over a region of axion masses from 1 to $100 \mathrm{meV}$.
\end{abstract}

Keywords: quantum vacuum; Casimir pressure; axion; non-Newtonian gravity

\section{Introduction}

Since the development of quantum field theory, it has been appreciated that the quantum vacuum is a fundamental type of physical reality which potentially contains all varieties of elementary particles and their interactions. Although an infinitely large energy density of zero-point oscillations of quantum fields (the so-called virtual particles) could be considered to be catastrophic [1], convenient self-consistent procedures have been elaborated on how to makeit equal to zero in the empty Minkowski space and take it into account when calculating the probabilities of arbitrary processes in the framework of the Standard Model. In doing so, the quantum vacuum is usually responsible for some part of the measured quantity (for instance, the Lamb shift), whereas the rest of it is determined by the ordinary (real) particles.

There is, however, one physical phenomenon, where the measured quantity is determined entirely by the quantum vacuum. This is the Casimir effect arising in the quantization volumes restricted by some material boundaries or in cosmological models with nontrivial topology [2-5]. An essence of this effect is that although the vacuum energy in restricted or topologically nontrivial volumes remains infinitely large, it becomes finite when subtracting the vacuum energy of empty topologically trivial Minkowski space. The negative derivative of the obtained finite vacuum energy with respect to the 
length parameter (either a separation between the boundary surfaces or a scale of the topologically nontrivial manifold) results in the Casimir force, which generalizes the familiar van der Waals force in the case of larger separations when one should take into consideration the effects of relativistic retardation [5].

Recently it was understood that the quantum vacuum may be responsible for an impressive phenomenon in nature, i.e., for acceleration of expansion of the Universe [6]. This can be explained by an impact of the energy density of quantum vacuum (which is often referred to as dark energy) corresponding to a nonzero renormalized value of the cosmological constant in the Einstein equations of general relativity theory [7].

Another subject is that at sufficiently short separations between the boundary surfaces, vacuum forces are stronger than Newtonian gravitation. In this case, they form a background for testing new physics, such as Yukawa-type corrections to Newton's law of gravitation arising due to exchange of light hypothetical scalar particles [8] or due to spontaneous compactification of extra spatial dimensions at the low-energy compactification scale [9]. Forces of this kind would alter the energy eigenstates of a neutron in the gravity potential of the Earth, and are searched for by a technique called Gravity Resonance Spectroscopy [10-12] by the $q$ BOUNCE collaboration. On the background of Casimir forces, one could also search for axions and other axion-like particles [13-20] which are considered as hypothetical constituents of dark matter. It is remarkable that taken together, dark energy and dark matter contribute for more than $95 \%$ of the energy of the Universe, leaving less than $5 \%$ to the forms of energy we are presently capable to observe directly [6].

Precise measurements of the Casimir force revealed a problem that the experimental data agree with theoretical predictions of the fundamental Lifshitz theory only under the condition that in calculations one disregards the relaxation properties of conduction electrons and the conductivity at a constant current for metallic and dielectric boundary surfaces, respectively (see review in $[5,21]$ and more modern experiments [22-27]). Theoretically, it was shown that an inclusion of the relaxation properties of conduction electrons and the conductivity at a constant current in computations results in a violation of the Nernst heat theorem for the Casimir entropy (see review in [5,21] and further results [28-31]). Taking into account that both the relaxation properties of conduction electrons for metals and the conductivity at a constant current for dielectrics are well studied really existing phenomena, there must be profound physical reasons for disregarding them in calculations of the Casimir force caused by the zero-point and thermal fluctuations of the electromagnetic field.

All precise experiments on measuring the Casimir interaction mentioned above have been performed in the sphere-plate geometry at surface separations below a micrometer. In this paper, we consider the CANNEX (Casimir And Non-Newtonian force EXperiment) that was designed to test the quantum vacuum in the configuration of two parallel plates at separations up to $15 \mu \mathrm{m}$ [32,33]. In addition to the already discussed possibility of testing the nature of dark energy [32], we consider here the potentialities of this experiment for searching thermal effects in the Casimir force, stronger constraints on Yukawa-type corrections to Newton's gravitational law, and on the coupling constants of axion-like particles. For this purpose, a modification in the measurement scheme is proposed, which allows simultaneous measurement of both the Casimir pressure and its gradient. It is shown that after making several improvements to the setup, the CANNEX test would be capable of performing the first observation of thermal effects in the Casimir interaction and to strengthen the presently available limits on Yukawa-type corrections to Newtonian gravity by up to a factor of 10 . Stronger limits could also be obtained on the coupling constants of axion-like particles to nucleons within a wide range of axion masses.

The paper is organized as follows. In Section 2 we briefly describe the experimental setup with two parallel plates and possible improvements. Section 3 is devoted to the calculation of thermal effects in the Casimir pressure and its gradient at separations relevant for CANNEX. In Section 4 the prospective constraints on non-Newtonian gravity and axion-like particles, which could be obtained from the improved setup, are found. Section 6 contains our conclusions and discussion. 


\section{Experimental Setup with Improved Precision}

We recently demonstrated the feasibility of Casimir pressure gradient measurements with the parallel plate CANNEX setup [33]. In the present article, we propose several improvements to the experiment that will lead to a significant increase in sensitivity and reduce the influence of systematic effects.

In our setup shown in Figure 1, the parallel plate geometry is implemented by a rigid vertical $\mathrm{SiO}_{2}$ cylinder (lower plate) of radius $R=5.742 \mathrm{~mm}$ separated by a vacuum gap of width $a$ from a movable (upper) sensor plate that can be described as a lumped mass-spring system [32]. This sensor with an interacting area $A$, elastic constant $k$, and effective mass $m_{\text {eff }}$ has a free resonance frequency $\omega_{0}=\sqrt{k / m_{\text {eff }}}$. In [33] an interferometric detection scheme was used to measure the gradient of the Casimir pressure. Here, we propose to implement the same scheme to measure both pressure gradients and the pressure acting between the two flat plates. Gradients $P^{\prime}(a) \equiv \partial P / \partial a$ are detected using a phase-locked loop (PLL) that senses shifts

$$
\Delta \omega=\sqrt{\omega_{0}^{2}-\frac{A}{m_{\mathrm{eff}}} \frac{\partial P}{\partial a}}-\omega_{0}
$$

of the sensor's resonance frequency—a technique widely used in the literature [34-38].

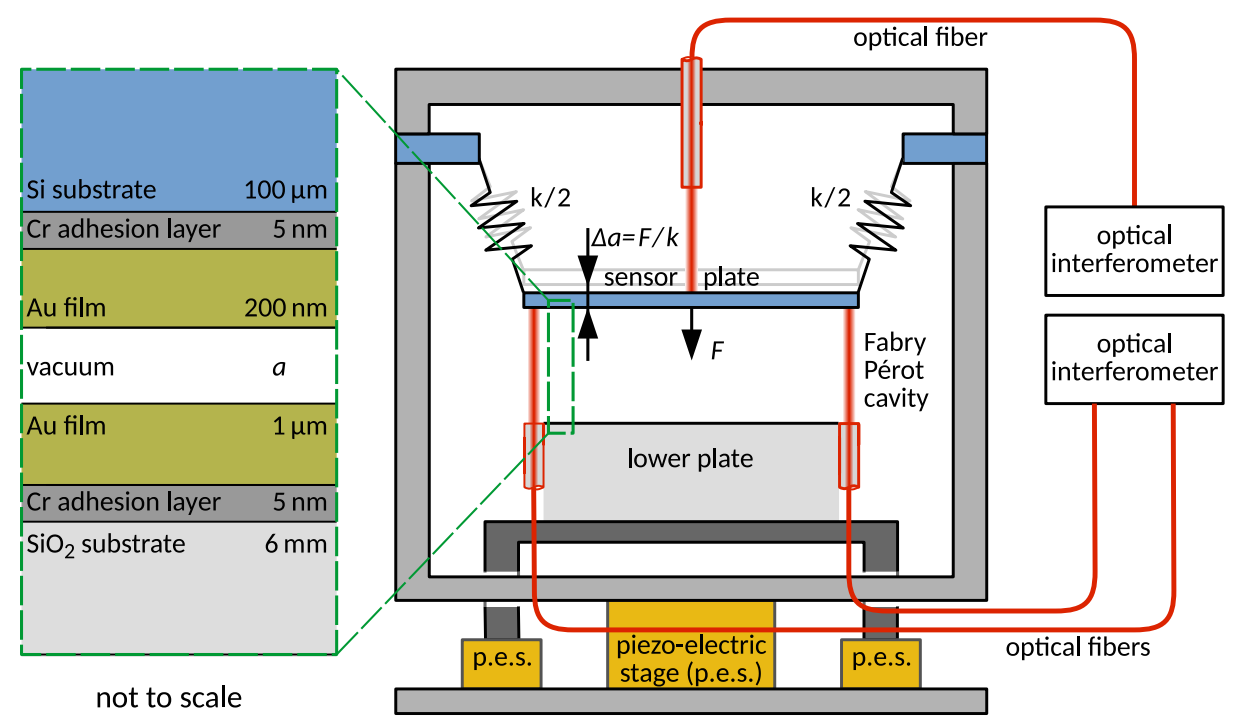

Figure 1. Simplified two-dimensional schematic representation of our setup with included improvements proposed in the main text. The material composition of the surfaces is shown separately on the left.

In the absence of thermal drifts, the pressure $P$ between our flat surfaces can be measured by monitoring the extension $\Delta a=P A / k$ of the spring-mass system using the same interferometer. For the determination and maintenance of parallelism, however, we recently used a feedback mechanism based on the capacitance between the two plates. While this scheme has been demonstrated to work in principle, practice has shown that vibrations in combination with the high $Q$-factor of our sensor lead to unacceptably long integration times, and associated susceptibility to thermal drifts [33]. To improve the performance and reach the full potential of the setup, we propose to replace the capacitive scheme by an interferometric one. As shown in Figure 1, three Fabry Pérot interferometers below the sensor plate (created by the end faces of optical fibers and the reflective surface of the sensor plate) monitor the distance $a$ at different positions around the lower plate. (Please note that in the two-dimensional scheme in the figure, only two of these cavities are depicted.) The three fiber ends can be polished together with the lower plate to exactly match the surface of the latter. In such a system, the three lower interferometers could measure the tilt and frequency shift at all times. Synchronously, the upper 
(fourth) interferometer, being mechanically connected to the sensor frame, can be used to measure the extension $\Delta a$ of the sensor and, hence, the pressure acting on it.

Besides this conceptual change several technical improvements are planned, which will result in the following: First, we aim to improve the seismic attenuation by a factor of 10 around $f_{0}=\omega_{0} /(2 \pi)$ with respect to the present performance by means of active control techniques [39]. This would not only reduce the direct influence on measurements via non-linear effects and rms noise, but would also improve the stability of various feedback circuits in a nontrivial way. Second, a newly designed distributed thermal control concept will guarantee $\mathrm{mK}$ stability throughout the setup, thereby eliminating drift and uncertainty in the sensor characteristics. Third, electrostatic patch effects that create a systematic pressure background could be reduced using in situ Ar ion bombardment [38]. In the following, we analyze the major sources of experimental uncertainty and show the predicted effect of the mentioned improvements.

\subsection{Sources of Error}

Here, we consider experimental errors in the pressure and its gradient due to vibrations, the determination of displacement, and frequency shift of our sensor, variations in the temperature, tilt of the plates, and discuss the role of electrostatic patch effects.

Previously, we identified vibrations as a major source of error and gave respective tolerable limits for pressure measurements [33]. Using a five-axis seismic attenuation system, we achieved a damping factor of 60 in vertical direction around the sensor resonance $f_{0}=10.24 \mathrm{~Hz}$. The residual vibrations caused at times a peak displacement noise $\delta a_{p} \approx 300 \mathrm{~nm}$ of the sensor relative to the lower plate, which proved to be a severe nuisance during the measurements. For pressure gradients, the main influence is via non-linear effects [34,40]. For a function $\phi(a)$ representing either the pressure $(\phi=P)$ or its gradient $(\phi=\partial P / \partial a)$, we expand

$$
\phi(a+\delta a) \approx \phi(a)+\langle\delta a\rangle \frac{\partial}{\partial a} \phi(a)+\frac{1}{2}\left\langle\delta a^{2}\right\rangle \frac{\partial^{2}}{\partial a^{2}} \phi(a)
$$

for small sensor movements $\delta a$. Recognizing that $\langle\delta a\rangle \equiv 0$, as usual for stochastic fluctuations, the main contribution to the error comes from the second order term. Thus, for the non-linear shifts in the pressure and its gradient, we therefore have

$$
\delta P_{\mathrm{nl}} \approx \frac{1}{2}\left\langle\delta a^{2}\right\rangle \frac{\partial^{2}}{\partial a^{2}} P(a), \text { and } \delta\left(\frac{\partial P}{\partial a}\right)_{\mathrm{nl}} \approx \frac{1}{2}\left\langle\delta a^{2}\right\rangle \frac{\partial^{3}}{\partial a^{3}} P(a),
$$

respectively. Similarly, the rms pressure and pressure gradient (entering measurements mainly as a nuisance within the mechanical bandwidth of the sensor $2 \delta f_{\mathrm{BW}}=2.8 \mathrm{mHz}$ around $f_{0}$ ) are computed from the spectral sensor movement $\delta a(f)$ via

$$
\delta \phi_{\mathrm{rms}} \approx \frac{\partial \phi(a)}{\partial a}\left[\int_{f_{0}-\delta f_{\mathrm{BW}}}^{f_{0}+\delta f_{\mathrm{BW}}} \mathrm{d} f \delta a^{2}(f)\right]^{1 / 2} .
$$

We note that vibrational noise also hampers the convergence of various feedback circuits and thereby influences the achievable sensitivity in a nontrivial way. Experience has shown that such effects are negligible below a peak amplitude of $\sim 20 \mathrm{~nm}$.

Another fundamental source of error is the uncertainty in various parameters involved in the evaluation of the frequency shift. We consider the following calibration procedure. At large separation $a_{c a l} \approx 80 \mu \mathrm{m}$ we apply the AC electrostatic excitation voltages $V_{\mathrm{ex}}$ and $V_{\mathrm{AC}}$ driving the sensor resonance and the surface potential compensation circuit, respectively [33]. At this separation, the Casimir pressure $P$ is negligibly small with respect to the electrostatic pressure $P_{\mathrm{ES}}$. The free resonance frequency 


$$
\omega_{0}=\left(\omega_{0 P}^{2}+\frac{A}{m_{\mathrm{eff}}}\left[\left.\frac{\partial P_{\mathrm{ES}}}{\partial a}\right|_{a_{\text {cal }}}+\left.\frac{\partial P}{\partial a}\right|_{a_{\mathrm{cal}}}\right]\right)^{1 / 2} \quad \text { with } \frac{\partial P_{\mathrm{ES}}}{\partial a}=\frac{\varepsilon_{0}}{2 a_{\mathrm{cal}}^{3}}\left(V_{\mathrm{AC}}^{2}+V_{\mathrm{ex}}^{2}\right)
$$

is determined from a measurement of the resonance frequency $\omega_{0 P}$ under the influence of the well-known electrostatic and Casimir forces. Here, $\varepsilon_{0}$ is the dielectric permittivity of vacuum. The effective mass $m_{\text {eff }}$ is determined during a separate sweep recording $\omega_{0 P}$ as a function of an applied DC electrostatic potential. Eventually, we evaluate the resonance frequency shift measured at different separations to obtain the Casimir pressure gradient from Equation (1) after subtraction of the electrostatic pressure gradient $\partial P_{\mathrm{ES}} / \partial a$. For pressure measurements at the same separations, we evaluate the detected extension $\Delta a$ of the sensor that is related to the total pressure $P_{\text {tot }}=P+P_{\mathrm{ES}}=$ $\Delta a \omega_{0}^{2} m / A$. The achievable sensitivity for both types of measurement is limited by the uncertainties in all experimental quantities entering the respective evaluation. Numerical values for these and some other uncertainties considered below are given in Table 1.

Variations in the temperature contribute in two ways to the experimental error. First, different material expansion coefficients influence the separation between the two interacting surfaces by roughly $63 \mathrm{~nm} / \mathrm{K}$-an effect most influential at smaller $a$. Second, the temperature influences the Youngs modulus of our sensor, which leads to an additional error in $a$, but also offsets the resonance frequency and, thereby, mimics a pressure gradient.

We also consider errors due to tilt of the plates with respect to each other. For small angles $\delta \alpha$ between the plates, we may estimate the influence on the pressure and its gradient by averaging

$$
\delta \phi(a, \delta \alpha) \approx \int_{A} \mathrm{~d} A \phi[a(x, y, \delta \alpha)]
$$

over the sensor area $A$ with the local separation $a(x, y, \delta \alpha)$ deviating from its nominal value $a$ due to the tilt. Numerical calculations show that the respective relative corrections to the pressure and its gradient are both of the order $(\delta \alpha R / a)^{2}$, in agreement with the literature [5]. For the achievable values of $\delta a$ given in Table 1 , these corrections are negligible.

As has been discussed in Ref. [33], electrostatic effects can have a significant influence. While we recently compared our measurements with the model [41], we now use the model [42] that has been shown to describe the observed forces more realistically. The average patch size $\langle\ell\rangle=0.82 \mu \mathrm{m}$ and the value for $\left\langle\ell^{2}\right\rangle$ mentioned in Table 1 were derived from the auto-correlation of actual Kelvin probe data for our surfaces. As $\langle\ell\rangle$ is much smaller than the plate separation, we can use the approximation

$$
P_{\text {patch }}(a) \approx \frac{3 \zeta(3) \varepsilon_{0} V_{\mathrm{rms}}^{2}\left\langle\ell^{2}\right\rangle}{4 a^{4}} .
$$

Table 1. Values for the experimental parameters and uncertainties for the setup in its present configuration and after the proposed improvements.

\begin{tabular}{lccc}
\hline Parameter & Present Value & Improved Value & \\
\hline Separation uncertainty $\delta a_{\text {cal }}$ & 2.0 & 0.5 & $\mathrm{~nm}$ \\
Mass calibration error $\delta m_{\text {eff }}$ & 1.0 & 0.01 & $\%$ \\
Area uncertainty $\delta A$ & $5 \times 10^{-4}$ & $5 \times 10^{-4}$ & $\mathrm{~cm}^{2}$ \\
Applied AC voltages uncertainty & 10 & 1 & $\mu \mathrm{V}$ \\
Frequency measurement error & $10^{-5}$ & $4 \times 10^{-7}$ & $\mathrm{~Hz}$ \\
Vibration amplitude at $f_{0}$ & 300 & 20 & $\mathrm{~nm}$ \\
Patches $V_{\mathrm{rms}}$ & 1.28 & 0.64 & $\mathrm{~V}$ \\
Patches $\left\langle\ell^{2}\right\rangle$ & 7.3 & 2.4 & $\mu \mathrm{m}^{2}$ \\
Tilt angle $\delta \alpha$ & 3.0 & 0.1 & $\mu \mathrm{rad}$ \\
Temperature stability & 3.0 & 0.5 & $\mathrm{mK}$ \\
\hline
\end{tabular}


Here, $V_{\mathrm{rms}}=0.634 \mathrm{mV}$, and $\zeta(z)$ is the Riemann zeta function. Please note that $P_{\text {patch }}$ represents a systematic effect that can be characterized and removed from experimental data.

\subsection{Sensitivity Estimation}

Based on the models described in Section 2.1, we have calculated the expected level of experimental uncertainty in measurements of the pressure and its gradient for both the present situation and presuming a successful implementation of the proposed improvements. For these calculations, we have assumed the parameters listed in Table 1. As can be seen in Figure 2a, the largest uncertainty in force measurements comes from the determination of the sensor extension, which includes contributions from the calibration and interferometry. Vibrations play, under the assumption that the proposed measures work as expected, a minor role. For force gradients (Figure 2b) the determination of the frequency shift is the limiting factor at separations larger than $\sim 4 \mu \mathrm{m}$, while at smaller separations sensitivity is limited by vibrations. Temperature variations are influential on force gradient measurements for all separations, as they modify the Youngs modulus of our sensor. For comparison, we also plot the achievable uncertainty for the present version of the setup (dashed upper red lines), which are determined by the same factors as for the improved version. Please note that the patch pressure can be characterized separately and removed from the data.
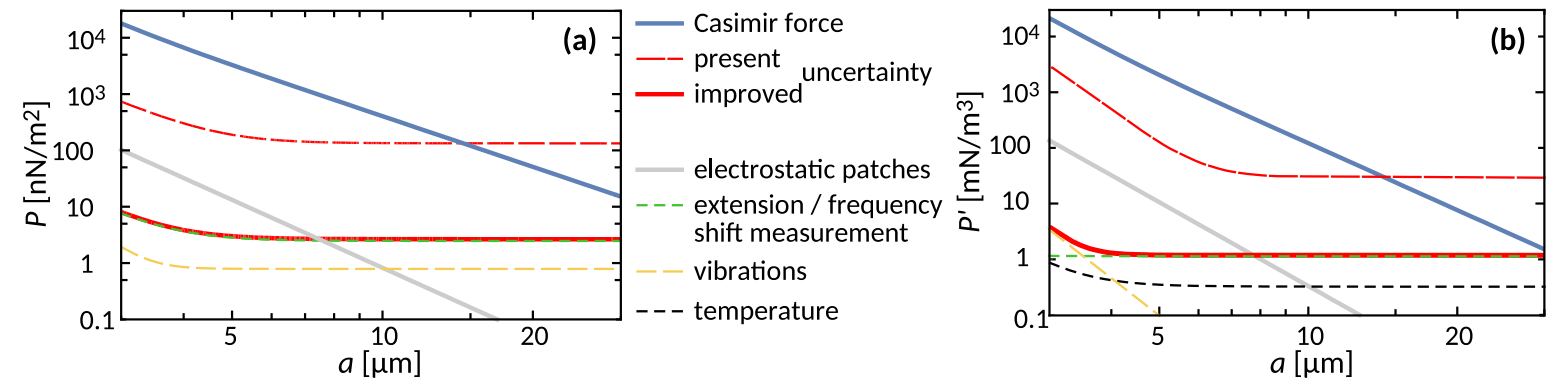

Figure 2. Estimated experimental uncertainties for the present and improved versions of the setup as a function of separation between the two parallel plates. (a) Pressure measurements using the upper interferometer. (b) Pressure gradient measurements using the lower interferometer.

The results for the improved uncertainties in Figure 2 are based on very conservative estimates. In the experiment, especially at larger separations, the uncertainty in the pressure could be reduced statistically by longer measurements. For further calculations in Section 4 , we therefore assume a pressure sensitivity of $1 \mathrm{nN} / \mathrm{m}^{2}$ (corresponding to $0.1 \mathrm{pN}$ ) at $a \geq 4 \mu \mathrm{m}$, which is slightly higher than the value corresponding to the bottom red line in Figure 2a. The uncertainty in the pressure gradient is mainly determined by the resolution of the PLL's frequency measurement. Here, the possibility for a statistical reduction of the uncertainty may be more problematic and therefore we use the pressure gradient sensitivity given by the bottom red line in Figure $2 \mathrm{~b}$ for calculations in Section 4 .

\section{Possibilities to Measure Thermal Effects in the Casimir Force}

As mentioned in Section 1, most of the already performed experiments on measuring the Casimir interaction exploited the sphere-plate geometry. In doing so, the measured quantity was either the Casimir force acting between a sphere and a plate (in the static measurement scheme) or its gradient (in the dynamic measurement scheme). Due to the proximity force approximation, the latter quantity can be recalculated into the effective Casimir pressure between two parallel plates $[5,21]$. There is only one modern experiment on the direct measurement of the Casimir pressure between two parallel plates [43], but it is not of sufficient precision to observe the thermal effects (an attempt to measure the Casimir effect between two parallel Al-coated plates at separations larger than a few micrometers was unsuccessful due to the presence of large background forces [44]). 
The distinctive feature of the CANNEX test of the quantum vacuum is that it can be adapted for simultaneous measurements of the Casimir pressure between two parallel plates and its gradient (see Section 2).

The Lifshitz formula for the Casimir pressure between two material plates spaced at a separation $a$ at temperature $T$ is given by $[5,21]$

$$
P(a)=-\frac{k_{B} T}{\pi} \sum_{l=0}^{\infty} \int_{0}^{\infty} k_{\perp} d k_{\perp} q_{l} \sum_{\alpha} \frac{r_{\alpha}^{2}\left(i \xi_{l}, k_{\perp}\right)}{e^{2 a q_{l}}-r_{\alpha}^{2}\left(i \xi_{l}, k_{\perp}\right)} .
$$

Here, it is assumed that the parallel plates made of a nonmagnetic material described by the dielectric permittivity $\varepsilon(\omega)$ are in thermal equilibrium with the environment at temperature $T$ and the following notations are introduced. The Boltzmann constant is $k_{B}$, the prime on the summation sign divides the term with $l=0$ by $2, k_{\perp}$ is the magnitude of the projection of the wave vector on the plane of the plates, $\xi_{l}=2 \pi k_{B} T l / \hbar$ with $l=0,1,2, \ldots$ are the Matsubara frequencies, and $q_{l}=\left(k_{\perp}^{2}+\xi_{l}^{2} / c^{2}\right)^{1 / 2}$.

The reflection coefficients $r_{\alpha}$ are defined for two independent polarizations of the electromagnetic field, transverse magnetic $(\alpha=\mathrm{TM})$ and transverse electric $(\alpha=\mathrm{TE})$. Explicitly they are given by

$$
r_{\mathrm{TM}}\left(i \xi_{l}, k_{\perp}\right)=\frac{\varepsilon_{l} q_{l}-k_{l}}{\varepsilon_{l} q_{l}+k_{l}}, \quad r_{\mathrm{TE}}\left(i \xi_{l}, k_{\perp}\right)=\frac{q_{l}-k_{l}}{q_{l}+k_{l}},
$$

where

$$
k_{l} \equiv k\left(i \xi_{l}, k_{\perp}\right)=\left(k_{\perp}^{2}+\varepsilon_{l} \frac{\xi_{l}^{2}}{c^{2}}\right)^{1 / 2}, \quad \varepsilon_{l} \equiv \varepsilon\left(i \xi_{l}\right) .
$$

By differentiating Equation (8) with respect to separation between the plates $a$, one obtains the gradient of the Casimir pressure

$$
P^{\prime}(a)=\frac{2 k_{B} T}{\pi} \sum_{l=0}^{\infty} \int_{0}^{\infty} k_{\perp} d k_{\perp} q_{l}^{2} \sum_{\alpha} \frac{r_{\alpha}^{2}\left(i \xi_{l}, k_{\perp}\right) e^{2 a q_{l}}}{\left[e^{2 a q_{l}}-r_{\alpha}^{2}\left(i \xi_{l}, k_{\perp}\right)\right]^{2}} .
$$

Equations (8) and (11) take an exact account of the effects of finite conductivity of the plate metal. As to corrections due to surface roughness, at separations exceeding $3 \mu \mathrm{m}$ they are much smaller than an error in the pressure measurements [5].

For numerical computations it is convenient to introduce the dimensionless variables

$$
y=2 a q_{l}, \quad \zeta_{l}=\frac{2 a \tilde{\zeta}_{l}}{c} .
$$

In terms of these variables Equations (8) and (11) take the form

$$
P(a)=-\frac{k_{B} T}{8 \pi a^{3}} \sum_{l=0}^{\infty} \int_{\zeta_{l}}^{\infty} y^{2} d y \sum_{\alpha} \frac{r_{\alpha}^{2}\left(i \zeta_{l}, y\right) e^{-y}}{1-r_{\alpha}^{2}\left(i \zeta_{l}, y\right) e^{-y}}
$$

and

$$
P^{\prime}(a)=\frac{k_{B} T}{8 \pi a^{4}} \sum_{l=0}^{\infty} \int_{\zeta_{l}}^{\infty} y^{3} d y \sum_{\alpha} \frac{r_{\alpha}^{2}\left(i \zeta_{l}, y\right) e^{-y}}{\left[1-r_{\alpha}^{2}\left(i \zeta_{l}, y\right) e^{-y}\right]^{2}},
$$

respectively, and the reflection coefficients from Equation (9) take the form

$$
r_{\mathrm{TM}}\left(i \zeta_{l}, y\right)=\frac{\varepsilon_{l} y-\sqrt{y^{2}+\left(\varepsilon_{l}-1\right) \zeta_{l}^{2}}}{\varepsilon_{l} y+\sqrt{y^{2}+\left(\varepsilon_{l}-1\right) \zeta_{l}^{2}}}, \quad r_{\mathrm{TE}}\left(i \zeta_{l}, y\right)=\frac{y-\sqrt{y^{2}+\left(\varepsilon_{l}-1\right) \zeta_{l}^{2}}}{y+\sqrt{y^{2}+\left(\varepsilon_{l}-1\right) \zeta_{l}^{2}}} .
$$


For application to the experimental setup of CANNEX described in Section 2, computations should be made for Au plates. The dielectric permittivity of Au along the imaginary frequency axis is obtained by means of the Kramers-Kronig relations using the available tabulated optical data for the complex index of refraction extrapolated down to zero frequency [5,21]. According to Section 1, this extrapolation can be made either by means of the Drude model taking into account the relaxation properties of conduction electrons or the plasma model disregarding these relaxation properties. In dimensionless variables, the dielectric permittivity of the Drude model along the imaginary frequency axis is given by

$$
\varepsilon_{l}^{(D)}=1+\frac{\tilde{\omega}_{p}^{2}}{\zeta_{l}\left(\zeta_{l}+\tilde{\gamma}\right)}
$$

where the dimensionless plasma frequency $\tilde{\omega}_{p}$ and relaxation parameter $\tilde{\gamma}$ are connected with the dimensional ones by $\tilde{\omega}_{p}=2 a \omega_{p} / c$ and $\tilde{\gamma}=2 a \gamma / c$. For Au the standard values $\hbar \omega_{p}=9.0 \mathrm{eV}$ and $\hbar \gamma=35 \mathrm{meV}$ are used here. The dielectric permittivity of the plasma model $\varepsilon_{l}^{(p)}$ is obtained from Equation (16) by putting $\tilde{\gamma}=0$. Please note that for computations at separations $a \geq 3 \mu \mathrm{m}$ performed below the optical data contribute negligibly small, so that the obtained gradients of the Casimir pressure are mostly determined by the extrapolations to lower frequencies.

Before presenting the computational results, we note that in the high-temperature limit $T \gg T_{\mathrm{cr}}=$ $\hbar c /\left(2 k_{B} a\right)$ all the terms in Equations (13) and (14) with $l \geq 1$ are exponentially small and both the Casimir pressure and its gradient are given predominantly by the terms with $l=0$. The zero-frequency term of the Lifshitz formula takes different forms depending on the extrapolation used. If the plasma model $\varepsilon_{l}^{(p)}$ is used for extrapolation, one obtains

$$
P_{(p)}(a)=-\frac{k_{B} T}{16 \pi a^{3}}\left[\int_{0}^{\infty} d y \frac{y^{2} e^{-y}}{1-e^{-y}}+\int_{0}^{\infty} d y \frac{y^{2} r_{\mathrm{TE}}^{2}(0, y) e^{-y}}{1-r_{\mathrm{TE}}^{2}(0, y) e^{-y}}\right]
$$

and

$$
P_{(p)}^{\prime}(a)=\frac{k_{B} T}{16 \pi a^{4}}\left[\int_{0}^{\infty} d y \frac{y^{3} e^{-y}}{\left(1-e^{-y}\right)^{2}}+\int_{0}^{\infty} d y \frac{y^{3} r_{\mathrm{TE}}^{2}(0, y) e^{-y}}{\left[1-r_{\mathrm{TE}}^{2}(0, y) e^{-y}\right]^{2}}\right],
$$

where in accordance to Equation (15)

$$
r_{\mathrm{TE}}(0, y)=\frac{y-\sqrt{y^{2}+\tilde{\omega}_{p}^{2}}}{y+\sqrt{y^{2}+\tilde{\omega}_{p}^{2}}} .
$$

Calculating the first integrals on the right-hand side of Equations (17) and (18) and expanding the second ones in the powers of a small parameter $1 / \tilde{\omega}_{p}$, we find [5]

$$
P_{(p)}(a)=-\frac{k_{B} T \zeta(3)}{4 \pi a^{3}}\left(1-\frac{6}{\tilde{\omega}_{p}}+\frac{48}{\tilde{\omega}_{p}^{2}}\right), \quad P_{(p)}^{\prime}(a)=\frac{3 k_{B} T \zeta(3)}{4 \pi a^{4}}\left(1-\frac{8}{\tilde{\omega}_{p}}+\frac{80}{\tilde{\omega}_{p}^{2}}\right) .
$$

If, however, the Drude model $\varepsilon_{l}^{(D)}$ is used for extrapolation, one arrives at the result

$$
P_{(D)}(a)=-\frac{k_{B} T}{16 \pi a^{3}} \int_{0}^{\infty} d y \frac{y^{2} e^{-y}}{1-e^{-y}}=-\frac{k_{B} T \zeta(3)}{8 \pi a^{3}}
$$

and

$$
P_{(D)}^{\prime}(a)=\frac{k_{B} T}{16 \pi a^{4}} \int_{0}^{\infty} d y \frac{y^{3} e^{-y}}{\left(1-e^{-y}\right)^{2}}=\frac{3 k_{B} T \zeta(3)}{8 \pi a^{4}} .
$$


Please note that at $T=300 \mathrm{~K}$ Equations (20)-(22) are applicable under a condition

$$
a \gg \frac{\hbar c}{2 k_{B} T} \approx 4 \mu \mathrm{m} .
$$

Taking into account, however, that the powers in all exponentially small terms with $l \geq 1$ depend on $-2 \pi T / T_{\text {cr }}$, one can see that at $T=300 \mathrm{~K}$ Equations (20)-(22) lead to rather precise results at all separations exceeding 6 or $7 \mu \mathrm{m}$.

The computational results for the magnitude of the Casimir pressure and its gradient as functions of separation between the plates are presented in Figure 3 by the red and blue solid lines computed at $T=300 \mathrm{~K}$ using extrapolations of the optical data of Au by means of the plasma and Drude models, respectively. For comparison purposes, the computational results at $T=0$ are presented by the dashed lines. They are obtained by Equations (13) and (14) where summation over the discrete Matsubara frequencies is replaced with a continuous integration. It is taken into account that with decreasing temperature the relaxation parameter $\gamma$ quickly decreases towards a very small residual value at $T=0$ that is determined by the defects of the crystal lattice. As a result, the values of $P(a)$ and $P^{\prime}(a)$ computed at $T=0$ using the plasma and Drude models coincide at high accuracy.
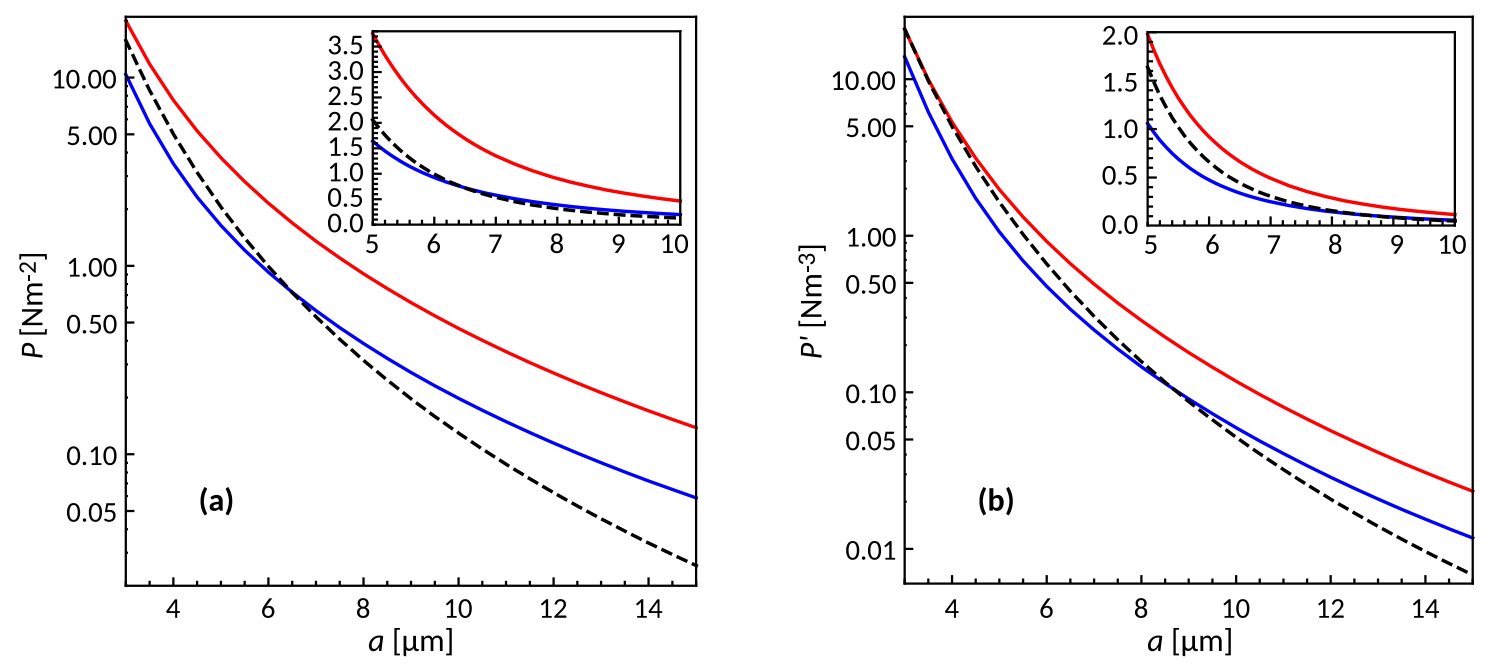

Figure 3. (a) The magnitude of the Casimir pressure between two parallel Au plates and (b) the pressure gradient are shown as functions of separation by the red and blue solid lines computed at $T=300 \mathrm{~K}$ using the plasma and Drude extrapolations of the optical data, respectively. The same quantities computed at $T=0$ are shown by the dashed lines. The region of separations from 5 to $10 \mu \mathrm{m}$ is shown in the insets using the homogeneous scale.

For a better visualization, in the insets to Figure $3 a, b$ the computational results in the separation region from 5 to $10 \mu \mathrm{m}$ are presented using a uniform scale on the vertical axis. It is clearly seen that the theoretical predictions from using the plasma and Drude model extrapolations of the optical data can be discriminated if to take into account the errors in measuring $P(a)$ and $P^{\prime}(a)$ in the improved version of CANNEX discussed in Section 2. Please note that if the Drude model extrapolation is used the thermal effect in the Casimir pressure and its gradient vanishes at approximately 6.4 and $8.7 \mu \mathrm{m}$ separations, respectively, where the blue and dashed lines intersect.

To determine the specific role of thermal effects in the Casimir pressure and its gradient, we have also computed the relative thermal corrections defined as

$$
\delta_{T} P(a)=\frac{P(a, T)-P(a, 0)}{P(a, 0)}, \quad \delta_{T} P^{\prime}(a)=\frac{P^{\prime}(a, T)-P^{\prime}(a, 0)}{P^{\prime}(a, 0)},
$$


where on the right-hand sides we indicated the dependence on temperature explicitly. Computational results for $\delta_{T} P$ and $\delta_{T} P^{\prime}$ are presented in Figure $4 \mathrm{a}, \mathrm{b}$ as functions of separation by the red and blue lines for the cases when extrapolation of the optical data for Au to lower frequencies is made by means of the plasma and Drude models, respectively. As is seen in these figures, within the separation range from 3 to $15 \mu \mathrm{m}$ the thermal effects make a considerable contribution to the Casimir pressure and its gradient. If the plasma model extrapolation is used, it reaches $440 \%$ and $240 \%$ of the zero-temperature pressure and its gradient, respectively, at $a=15 \mu \mathrm{m}$. When using the extrapolation by means of the Drude model, the relative thermal effect in the Casimir pressure varies from approximately $-34 \%$ at $a=3 \mu \mathrm{m}$ to $130 \%$ at $a=15 \mu \mathrm{m}$ and in the pressure gradient from approximately $-40 \%$ at $a=4 \mu \mathrm{m}$ to $60 \%$ at $a=15 \mu \mathrm{m}$. In this case, the thermal effect in the Casimir pressure vanishes at $a \approx 6.4 \mu \mathrm{m}$ and in its gradient at $a \approx 8.7 \mu \mathrm{m}$ in agreement with Figure $3 \mathrm{a}, \mathrm{b}$, respectively .
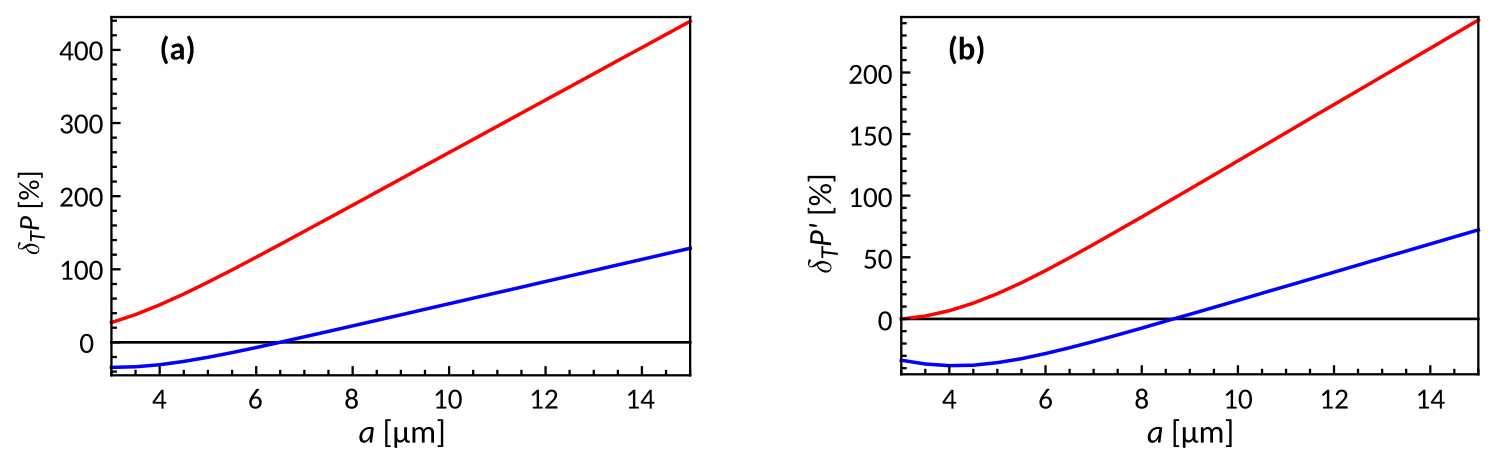

Figure 4. The relative thermal corrections to (a) the Casimir pressure between two parallel Au plates and (b) its gradient is shown as functions of separation by the red and blue lines computed at $T=300 \mathrm{~K}$ using the plasma and Drude extrapolations of the optical data, respectively.

\section{Prospective Constraints on Non-Newtonian Gravity and Axion-Like Particles}

As mentioned in Section 1, the Casimir forces originating from the quantum vacuum form a background for testing the Yukawa-type corrections to Newton's gravitational law and for searching the axion-like particles. It is convenient to parametrize the Yukawa-type potential between two point masses $m_{1}$ and $m_{2}$ situated at the points $\boldsymbol{r}_{1}$ and $\boldsymbol{r}_{2}$ as [8]

$$
V_{\mathrm{Yu}}(r) \equiv V_{\mathrm{Yu}}\left(\left|\boldsymbol{r}_{1}-\boldsymbol{r}_{2}\right|\right)=-\alpha \frac{G m_{1} m_{2}}{\left|\boldsymbol{r}_{1}-\boldsymbol{r}_{2}\right|} \exp \left(-\frac{\left(\left|\boldsymbol{r}_{1}-\boldsymbol{r}_{2}\right|\right.}{\lambda}\right) .
$$

Here, $\alpha$ and $\lambda$ are the interaction constant and the range of Yukawa interaction, and $G=6.674 \times 10^{-11} \mathrm{~m}^{3} \mathrm{~kg}^{-1} \mathrm{~s}^{-2}$ is the Newtonian gravitational constant (we note that in the experimental configuration under consideration one can neglect by the Newtonian gravitational pressure because it is less than an error in measurements of the Casimir pressure).

The Yukawa-type pressure between the top and bottom plates in the experimental setup of Section 2 should be calculated taking into account the layer structure of both plates shown in Figure 1. The top plate is made of high-resistivity $\mathrm{Si}$ of density $\rho_{\mathrm{Si}}=2.33 \mathrm{~g} \mathrm{~cm}^{-3}$ coated with a layer of Cr of density $\rho_{\mathrm{Cr}}=7.15 \mathrm{~g} \mathrm{~cm}^{-3}$ and thickness $d_{\mathrm{Cr}}=5 \mathrm{~nm}$ followed by a layer of Au of density $\rho_{\mathrm{Au}}=19.3 \mathrm{~g} \mathrm{~cm}^{-3}$ and thickness $d_{\mathrm{Au}}^{t}=200 \mathrm{~nm}$. The thickness of Si substrate $(100 \mu \mathrm{m})$ is sufficiently large to treat it as a semispace. The bottom plate is made of $\mathrm{SiO}_{2}$ quartz crystal with the density $\rho_{q}=2.64 \mathrm{~g} \mathrm{~cm}^{-3}$ coated with a layer of Cr of thickness $d_{\mathrm{Cr}}=5 \mathrm{~nm}$ followed by a layer of Au of thickness $d_{\mathrm{Au}}^{b}=1 \mu \mathrm{m}$. The thickness of $\mathrm{SiO}_{2}$ substrate $(6 \mathrm{~mm})$ again allows to consider it as a semispace.

Now we assume that one mass $\rho d V_{1}$ belongs to the top plate and another one $\rho d V_{2}$ to the bottom one and integrate Equation (25) over the volumes of both parallel plates separated by a distance $a$ 
taking into account their layer structure. Calculating the negative derivative of the obtained interacting energy with respect to $a$, we find the Yukawa force and finally the pressure [45]

$$
P_{\text {Yu }}(a)=-2 \pi G \alpha \lambda^{2} e^{-a / \lambda} \Phi(\lambda),
$$

where the function $\Phi$ is defined as

$$
\begin{aligned}
\Phi(\lambda)= & {\left[\rho_{\mathrm{Au}}-\left(\rho_{\mathrm{Au}}-\rho_{\mathrm{Cr}}\right) \exp \left(-\frac{d_{\mathrm{Au}}^{t}}{\lambda}\right)-\left(\rho_{\mathrm{Cr}}-\rho_{\mathrm{Si}}\right) \exp \left(-\frac{d_{\mathrm{Au}}^{t}+d_{\mathrm{Cr}}}{\lambda}\right)\right] } \\
& \times\left[\rho_{\mathrm{Au}}-\left(\rho_{\mathrm{Au}}-\rho_{\mathrm{Cr}}\right) \exp \left(-\frac{d_{\mathrm{Au}}^{b}}{\lambda}\right)-\left(\rho_{\mathrm{Cr}}-\rho_{q}\right) \exp \left(-\frac{d_{\mathrm{Au}}^{b}+d_{\mathrm{Cr}}}{\lambda}\right)\right] .
\end{aligned}
$$

The gradient of the Yukawa pressure is obtained by differentiating Equation (26) with respect to $a$

$$
P_{\mathrm{Yu}}^{\prime}(a)=2 \pi G \alpha \lambda e^{-a / \lambda} \Phi(\lambda)
$$

Now the constraints on the parameters $\alpha$ and $\lambda$ of Yukawa-type interactions can be obtained from the inequalities

$$
\left|P_{\mathrm{Yu}}(a)\right|<\Delta P(a), \quad P_{\mathrm{Yu}}^{\prime}(a)<\Delta P^{\prime}(a),
$$

where $\Delta P$ and $\Delta P^{\prime}$ are the total experimental errors in the measured Casimir pressure and its gradient estimated in Section 2. The meaning of Equation (29) is that the experimental data are found in agreement with theoretical predictions for the Casimir pressure and its gradient and no extra contribution of unknown origin was observed.

To estimate the strength of prospective constraints, which can be obtained from the improved version of CANNEX, we use the thickness of the top and bottom Au layers $d_{\mathrm{Au}}^{t}=200 \mathrm{~nm}$ and $d_{\mathrm{Au}}^{b}=1 \mu \mathrm{m}$ and the total experimental errors $\Delta P(a)=1 \mathrm{nN} / \mathrm{m}^{2}$ and $\Delta P^{\prime}(a)$ corresponding to the bottom red line in Figure 2b. The computational results for $\alpha$ and $\lambda$ obtained by using the first and second inequalities in Equation (29) are shown by the red lines in Figure 5a,b, respectively. In so doing, the regions of $(\alpha, \lambda)$-planes above each line are excluded and below each line are allowed.

For comparison purposes, we present in Figure $5 \mathrm{a}, \mathrm{b}$ the strongest constraints obtained in the same interaction range from other experiments. The top and bottom blue lines demonstrate the constraints found from the results of the Casimir-less experiment [46] and its improved version [47], respectively. Both experiments were performed by means of a micromechanical torsional oscillator. The green line shows the constraints obtained from measuring the difference in lateral forces [48]. The constraints indicated by the black line are found in [49] from the torsion pendulum experiment. Finally, the constraints of the gray line follow from the Cavendish-type experiments performed at short separations [50-52].

As is seen in Figure $5 \mathrm{a}, \mathrm{b}$, the strongest constraints obtained up to date follow from the improved Casimir-less experiment [47] (the bottom blue line). It is seen also that the largest strengthening of these constraints, which could be reached from the CANNEX test of quantum vacuum, follows from measurements of the Casimir pressure (this is because the pressure gradient is linear in $\lambda$ whereas the pressure is quadratic in $\lambda$ ). The prospective constraints are stronger by up to a factor of 10 over a wide interaction range.

Next we consider the prospective constraints on the axion-to-nucleon coupling constants which could be obtained from the CANNEX test of the quantum vacuum. Taking into account that the exchange of one axion between two nucleons results in the spin-dependent interaction potential [53] and the test bodies in this experiment are not polarized, any additional force of the axion origin could arise due to two-axion exchange. 
Below we deal with axion-like particles coupled to nucleons by means of pseudo-scalar interaction Lagrangian [54]. In this case, the effective potential between two nucleons, spaced at the points $r_{1}$ and $r_{2}$ of the top and bottom plates, arising due to exchange of two axions, takes the form [53,55]

$$
V_{a n}(r) \equiv V_{a n}\left(\left|\boldsymbol{r}_{1}-\boldsymbol{r}_{2}\right|\right)=-\frac{g_{a n}^{4}}{32 \pi^{3}} \frac{\hbar^{2} m_{a}}{m^{2}}\left(\boldsymbol{r}_{1}-\boldsymbol{r}_{2}\right)^{-2} K_{1}\left(\frac{2 m_{a} c\left|\boldsymbol{r}_{1}-\boldsymbol{r}_{2}\right|}{\hbar}\right) .
$$

Here, $g_{a n}$ is the dimensionless coupling constant of an axion to a nucleon (we assume that the coupling constants to a neutron and a proton are equal [53]), the mean of the proton and neutron masses is denoted as $m$, the axion mass is $m_{a}, K_{1}(z)$ is the modified Bessel function of the second kind and it is assumed that $r \gg \hbar /(m c)$.
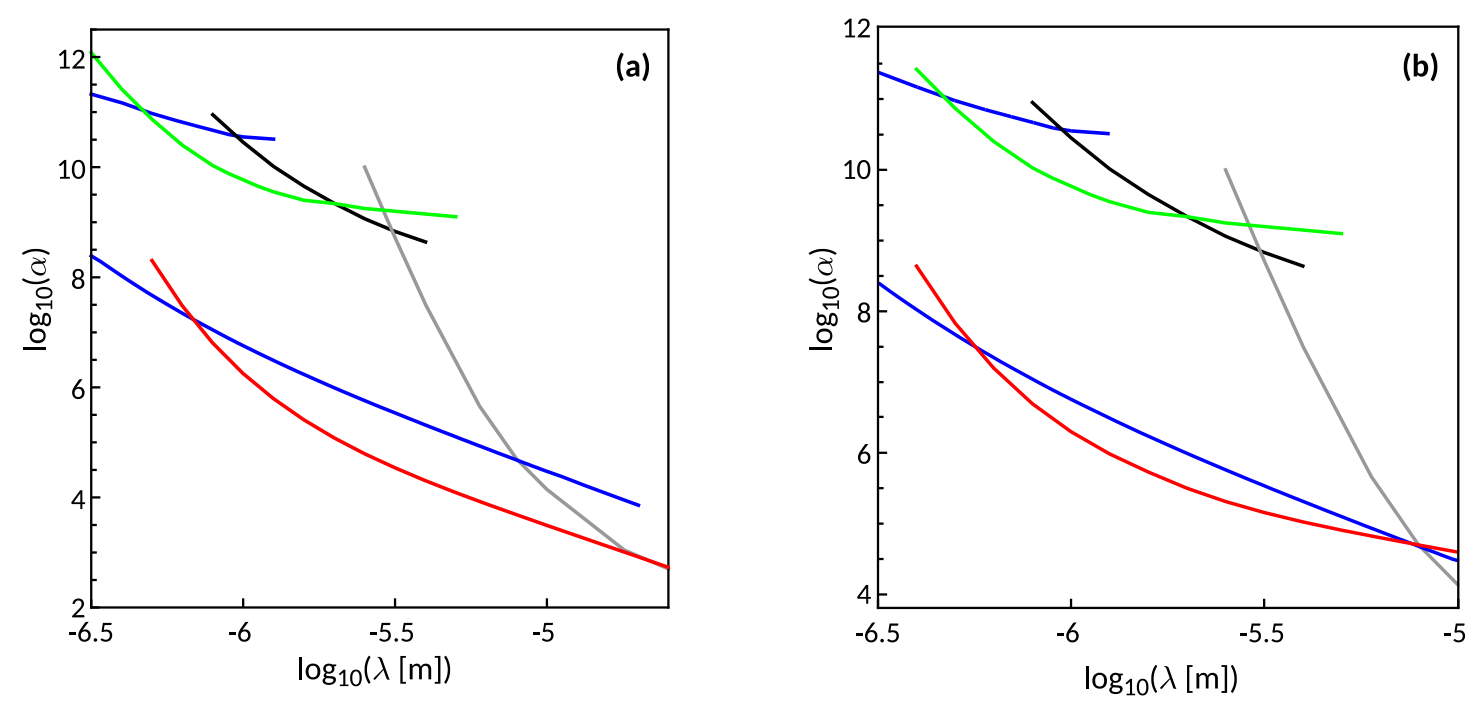

Figure 5. Constraints on the parameters of Yukawa-type interaction obtained from two Casimir-less experiments, from measuring the difference in lateral forces, from the torsion pendulum experiment and from the Cavendish-type experiments are shown by the top and bottom blue lines, green, black and gray lines, respectively. The proposed constraints which could be obtained from the CANNEX test of the quantum vacuum when measuring (a) the Casimir pressure and (b) its gradient are indicated by the red lines.

Similar to the case of a Yukawa-type potential, the additional pressure between the test bodies due to two-axion exchange, is obtained by integrating Equation (30) over the volumes of both plates with account of their layer structure, calculate the negative derivative of the obtained result with respect to $a$ and finally find the pressure (see [15] for details)

$$
P_{a n}(a)=-\frac{\hbar^{3}}{2 m^{2} m_{\mathrm{H}}^{2} c} \int_{1}^{\infty} d u \frac{\sqrt{u^{2}-1}}{u^{2}} \Psi\left(\frac{m_{a} c u}{\hbar}\right) \exp \left(-\frac{2 m_{a} c a u}{\hbar}\right) .
$$

Here, $m_{\mathrm{H}}$ is the mass of atomic hydrogen and the function $\Psi(x)$ is defined as

$$
\begin{aligned}
& \Psi(x)=\left[C_{\mathrm{Au}}\left(1-e^{-2 x d_{\mathrm{Au}}^{t}}\right)+C_{\mathrm{Cr}} e^{-2 x d_{\mathrm{Au}}^{t}}\left(1-e^{-2 x d_{\mathrm{Cr}}}\right)+C_{\mathrm{Si}} e^{-2 x\left(d_{\mathrm{Au}}^{t}+d_{\mathrm{Cr}}\right)}\right] \\
& \times\left[C_{\mathrm{Au}}\left(1-e^{-2 x d_{\mathrm{Au}}^{b}}\right)+C_{\mathrm{Cr}} e^{-2 x d_{\mathrm{Au}}^{b}}\left(1-e^{-2 x d_{\mathrm{Cr}}}\right)+C_{q} e^{-2 x\left(d_{\mathrm{Au}}^{b}+d_{\mathrm{Cr}}\right)}\right],
\end{aligned}
$$

where the coefficients $C$ for each material are given by

$$
C=\rho \frac{g_{a n}^{2}}{4 \pi}\left(\frac{Z}{\mu}+\frac{N}{\mu}\right) .
$$


In Equation (33), $\rho$ is the density of the respective material already indicated above, $Z$ and $N$ are the number of protons and the mean number of neutrons in the atoms of materials with mean mass $M$ and $\mu=M / m_{\mathrm{H}}$. For materials under consideration $Z / \mu=0.40422,0.46518,0.50238$, and 0.503205 for $\mathrm{Au}, \mathrm{Cr}, \mathrm{Si}$, and $\mathrm{SiO}_{2}$, respectively [8]. The values of $N / \mu$ are $0.60378,0.54379,0.50628$, and 0.505179 for the same respective materials [8].

The gradient of the pressure due to two-axion exchange is obtained from Equation (31) by the differentiation with respect to $a$

$$
P_{a n}^{\prime}(a)=\frac{m_{a} \hbar^{2}}{m^{2} m_{\mathrm{H}}^{2}} \int_{1}^{\infty} d u \frac{\sqrt{u^{2}-1}}{u} \Psi\left(\frac{m_{a} c u}{\hbar}\right) \exp \left(-\frac{2 m_{a} c a u}{\hbar}\right) .
$$

The constraints on the parameters of axion-nucleon interaction, $g_{a n}, m_{a}$, are obtained from the inequalities

$$
\left|P_{a n}(a)\right|<\Delta P(a), \quad P_{a n}^{\prime}(a)<\Delta P^{\prime}(a),
$$

similar to Equation (29).

To find the strongest prospective constraints on the axion-to-nucleon interaction, we have used the same parameters of the improved setup, as listed above when considering the Yukawa interaction. The computational results for $g_{a n}^{2} /(2 \pi)$ as a function of $m_{a} c^{2}$ obtained by using the first and second inequalities in Equation (35) are shown by the red lines in Figure $6 a, b$, respectively. Again, the space above each line is excluded (will be excluded, in the case of the red line) by the results of the respective experiment.

The strongest laboratory constraints obtained up to date [17] at the same region of axion masses are shown by the blue line. They follow from the improved Casimir-less experiment [47]. For comparison purposes, the constraints following from the Cavendish-type experiment [56,57], measurements of the effective Casimir pressure [15,58,59], and the lateral Casimir force between corrugated surfaces $[16,60,61]$ are shown by the gray, black, and green lines, respectively. As can be seen in Figure $6 \mathrm{a}, \mathrm{b}$, the stronger constraints again follow from measuring the Casimir pressure. According to Figure $6 \mathrm{a}$, the CANNEX test of the quantum vacuum could strengthen the presently known constraints by up to a factor of 3 .
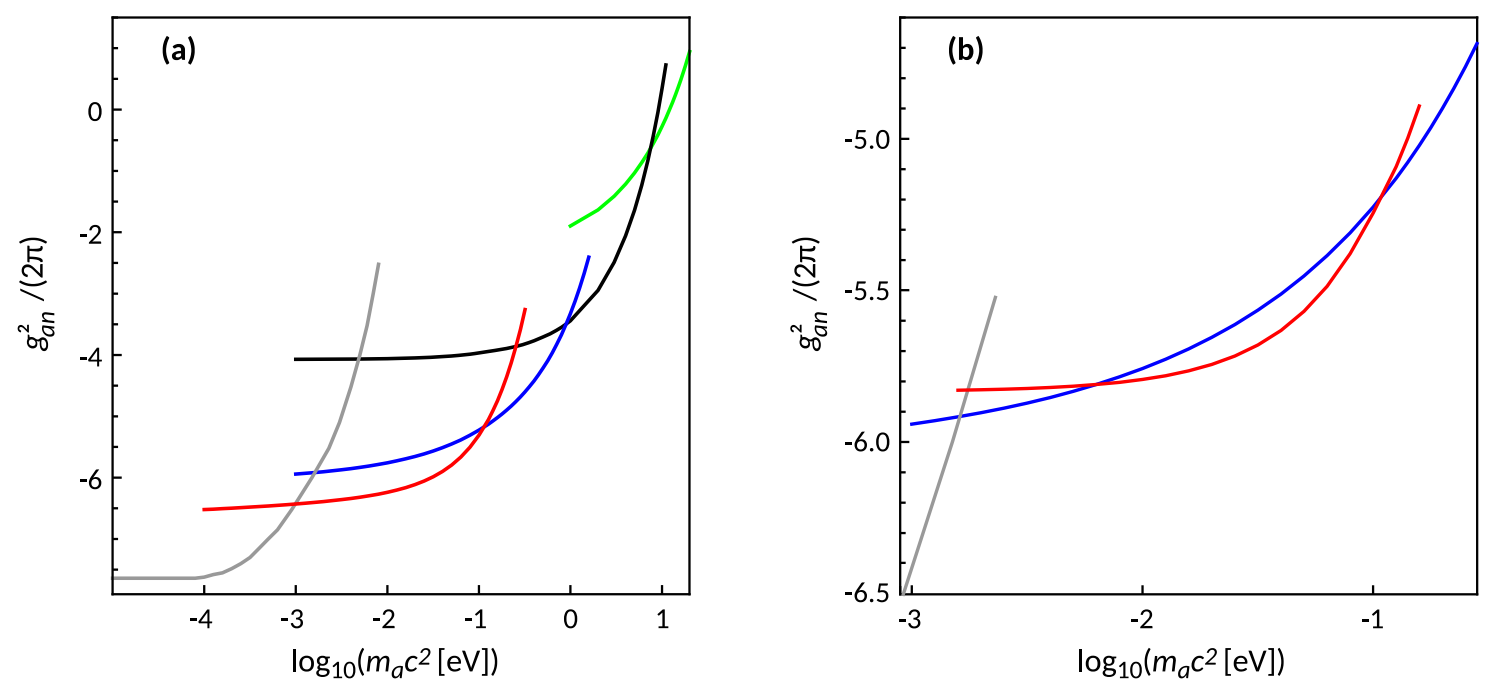

Figure 6. Constraints on the axion-to-nucleon coupling constant obtained from the improved Casimir-less experiment, the Cavendish-type experiment, measurements of the effective Casimir pressure, and the lateral Casimir force are shown by the blue, gray, black, and green lines, respectively. The proposed constraints which could be obtained from the CANNEX test of the quantum vacuum when measuring (a) the Casimir pressure and (b) its gradient are indicated by the red lines. 


\section{Discussion}

In the foregoing, we have considered the CANNEX test of the quantum vacuum intended to measure the gradient of the Casimir pressure between two parallel plates at separations exceeding a few micrometers. As already discussed in the literature [32,33], this experiment would be capable to place stronger constraints on the chameleon model of dark energy and, thus, bring new information concerning the origin of vacuum energy and the value of the cosmological constant. Here, we proposed a modification of the CANNEX setup, which allows for simultaneous measurements of both the pressure and its gradient. We also considered several improvements in the already existing setup which will allow for a more precise determination of several parameters. According to our results, with these improvements the CANNEX test of the quantum vacuum could be used to directly measure for the first time the thermal effects in the Casimir pressure and its gradient at separations from 5 to 10 micrometers. We have also shown that this experiment could strengthen the presently known best constraints on the parameters of non-Newtonian gravity by up to a factor of 10 over a wide interaction range. The constraints on the axion-to-nucleon coupling constant could be strengthened by up to a factor of 3 in the region of axion masses from 1 to $100 \mathrm{meV}$.

\section{Conclusions}

To conclude, CANNEX can be considered as a promising new laboratory experiment for investigating unusual features of the quantum vacuum, thermal effects in the Casimir forces, non-Newtonian gravity and properties of axion-like particles being hypothetical constituents of dark matter. Together with other laboratory experiments, such as demonstration of the dynamical Casimir effect in superconducting circuits [62,63], the large-scale accelerator projects, and astrophysical data, it may serve as the useful tool in resolving fundamental problems of the quantum vacuum.

Author Contributions: Investigation, G.L.K., V.M.M., R.I.P.S., and H.A.; writing, G.L.K., V.M.M., and R.I.P.S.; project administration, H.A.

Funding: This research was partially funded by the Russian Foundation for Basic Research grant number 19-02-00453 A.

Acknowledgments: V.M.M. was partially supported by the Russian Government Program of Competitive Growth of Kazan Federal University. G.L.K. and V.M.M. are grateful to the Atominstitut of the TU Wien, where this work was performed, for kind hospitality. R.I.P.S. was supported by the TU Wien.

Conflicts of Interest: The authors declare no conflict of interest.

\section{References}

1. Adler, R.J.; Casey, B.; Jacob, O.C. Vacuum catastrophe: An elementary exposition of the cosmological constant problem. Am. J. Phys. 1995, 63, 620-626. [CrossRef]

2. Elizalde, E. Ten Physical Applications of Spectral Zeta Functions, 2nd ed.; Springer-Verlag: Berlin, Germany, 2012.

3. Elizalde, E. The vacuum energy density for spherical and cylindrical universes. J. Math. Phys. 1994, 35, 3308-3321. [CrossRef]

4. Elizalde, E.; Nojiri, S.; Odintsov, S.D.; Ogushi, S. Casimir effect in de Sitter and anti-de Sitter braneworlds. Phys. Rev. D 2003, 67, 063515. [CrossRef]

5. Bordag, M.; Klimchitskaya, G.L.; Mohideen, U.; Mostepanenko, V.M. Advances in the Casimir Effect; Oxford University Press: Oxford, UK, 2015.

6. Frieman, J.; Turner, M.; Huterer, D. Dark Energy and the Accelerating Universe. Ann. Rev. Astron. Astrophys. 2008, 46, 385-432. [CrossRef]

7. Mostepanenko, V.M., Klimchitskaya, G.L. Whether an Enormously Large Energy Density of the Quantum Vacuum Is Catastrophic. Symmetry 2019, 11, 314. [CrossRef]

8. Fischbach, E.; Talmadge, C.L. The Search for Non-Newtonian Gravity; Springer-Verlag: New York, NY, USA, 1999.

9. Antoniadis, I.; Arkani-Hamed, N.; Dimopoulos, S.; Dvali, G. New dimensions at a millimeter to a fermi and superstrings at a TeV. Phys. Lett. B 1998, 436, 257-263. [CrossRef] 
10. Cronenberg, G.; Brax, P.; Filter, H.; Geltenbort, P.; Jenke, T.; Pignol, G.; Pitschmann, M.; Thalhammer, M.; Abele, H. Acoustic Rabi oscillations between gravitational quantum states and impact on symmetron dark energy. Nat. Phys. 2018, 14, 1022-1026. [CrossRef]

11. Jenke, T.; Cronenberg, G.; Burgdörfer, J.; Chizhova, L.A.; Geltenbort, P.; Ivanov, A.N.; Lauer, T.; Lins, T.; Rotter, S.; Saul, H.; et al. Gravity Resonance Spectroscopy Constrains Dark Energy and Dark Matter Scenarios. Phys. Rev. Lett. 2014, 112, 151105. [CrossRef]

12. Jenke, T.; Geltenbort, P.; Lemmel, H.; Abele, H. Realization of a gravity-resonance-spectroscopy technique. Nat. Phys. 2011, 7, 468-472. [CrossRef]

13. Bezerra, V.B.; Klimchitskaya, G.L.; Mostepanenko, V.M.; Romero, C. Constraints on the parameters of an axion from measurements of the thermal Casimir-Polder force. Phys. Rev. D 2014, 89, 035010. [CrossRef]

14. Bezerra, V.B.; Klimchitskaya, G.L.; Mostepanenko, V.M.; Romero, C. Stronger constraints on an axion from measuring the Casimir interaction by means of a dynamic atomic force microscope. Phys. Rev. D 2014, 89, 075002. [CrossRef]

15. Bezerra, V.B.; Klimchitskaya, G.L.; Mostepanenko, V.M.; Romero, C. Constraining axion-nucleon coupling constants from measurements of effective Casimir pressure by means of micromachined oscillator. Eur. Phys. J. C 2014, 74, 2859. [CrossRef]

16. Bezerra, V.B.; Klimchitskaya, G.L.; Mostepanenko, V.M.; Romero, C. Constraints on axion-nucleon coupling constants from measuring the Casimir force between corrugated surfaces. Phys. Rev. D 2014, 90, 055013. [CrossRef]

17. Klimchitskaya, G.L.; Mostepanenko, V.M. Improved constraints on the coupling constants of axion-like particles to nucleons from recent Casimir-less experiment. Eur. Phys. J. C 2015, 75, 164. [CrossRef]

18. Bezerra, V.B.; Klimchitskaya, G.L.; Mostepanenko, V.M.; Romero, C. Constraining axion coupling constants from measuring the Casimir interaction between polarized test bodies. Phys. Rev. D 2016, 94, 035011. [CrossRef]

19. Klimchitskaya, G.L. Recent breakthrough and outlook in constraining the non-Newtonian gravity and axion-like particles from Casimir physics. Eur. Phys. J. C 2017, 77, 315. [CrossRef]

20. Klimchitskaya, G.L.; Mostepanenko, V.M. Constraints on axionlike particles and non-Newtonian gravity from measuring the difference of Casimir forces. Phys. Rev. D 2017, 95, 123013. [CrossRef]

21. Klimchitskaya, G.L.; Mohideen, U.; Mostepanenko, V.M. The Casimir force between real materials: Experiment and theory. Rev. Mod. Phys. 2009, 81, 1827-1885. [CrossRef]

22. Banishev, A.A.; Chang, C.C.; Klimchitskaya, G.L.; Mostepanenko, V.M.; Mohideen, U. Measurement of the gradient of the Casimir force between a nonmagnetic gold sphere and a magnetic nickel plate. Phys. Rev. B 2012, 85, 195422. [CrossRef]

23. Banishev, A.A.; Klimchitskaya, G.L.; Mostepanenko, V.M.; Mohideen, U. Demonstration of the Casimir Force between Ferromagnetic Surfaces of a Ni-Coated Sphere and a Ni-Coated Plate. Phys. Rev. Lett. 2013, 110, 137401. [CrossRef]

24. Banishev, A.A.; Klimchitskaya, G.L.; Mostepanenko, V.M.; Mohideen, U. Casimir interaction between two magnetic metals in comparison with nonmagnetic test bodies. Phys. Rev. B 2013, 88, 155410. [CrossRef]

25. Bimonte, G.; López, D.; Decca, R.S. Isoelectronic determination of the thermal Casimir force. Phys. Rev. $B$ 2016, 93, 184434. [CrossRef]

26. Chang, C.C.; Banishev, A.A.; Klimchitskaya, G.L.; Mostepanenko, V.M.; Mohideen, U. Reduction of the Casimir Force from Indium Tin Oxide Film by UV Treatment. Phys. Rev. Lett. 2011, 107, 090403. [CrossRef]

27. Banishev, A.A.; Chang, C.C.; Castillo-Garza, R.; Klimchitskaya, G.L.; Mostepanenko, V.M.; Mohideen, U. Modifying the Casimir force between indium tin oxide film and Au sphere. Phys. Rev. B 2012, 85, 045436. [CrossRef]

28. Klimchitskaya, G.L.; Korikov, C.C. Casimir entropy for magnetodielectrics. J. Phys. Condens. Matter 2015, 27, 214007. [CrossRef] [PubMed]

29. Klimchitskaya, G.L.; Korikov, C.C. Analytic results for the Casimir free energy between ferromagnetic metals. Phys. Rev. A 2015, 91, 032119. [CrossRef]

30. Klimchitskaya, G.L.; Mostepanenko, V.M. Low-temperature behavior of the Casimir free energy and entropy of metallic films. Phys. Rev. A 2017, 95, 012130. [CrossRef]

31. Klimchitskaya, G.L.; Mostepanenko, V.M. Casimir free energy of dielectric films: classical limit, low-temperature behavior and control. J. Phys. Condens. Matter 2017, 29, 275701. [CrossRef] 
32. Almasi, A.; Brax, P.; Iannuzzi, D.; Sedmik, R.I.P. Force sensor for chameleon and Casimir force experiments with parallel-plate configuration. Phys. Rev. D 2015, 91, 102002. [CrossRef]

33. Sedmik, R.; Brax, P. Status Report and first Light from Cannex : Casimir Force Measurements between flat parallel Plates. J. Phys. Conf. Ser. 2018, 1138, 012014. [CrossRef]

34. Albrecht, T.R.; Grütter, P.; Horne, D.; Rugar, D. Frequency modulation detection using high- $Q$ cantilevers for enhanced force microscope sensitivity. J. Appl. Phys. 1991, 69, 668-673. [CrossRef]

35. Decca, R.S.; Fischbach, E.; Klimchitskaya, G.L.; Krause, D.E.; López, D.; Mostepanenko, V.M. Improved tests of extra-dimensional physics and thermal quantum field theory from new Casimir force measurements. Phys. Rev. D 2003, 68, 116003. [CrossRef]

36. Jourdan, G.; Lambrecht, A.; Comin, F.; Chevrier, J. Quantitative Non-Contact Dynamic Casimir Force Measurements. Europhys. Lett. 2009, 85, 31001. [CrossRef]

37. Chang, C.C.; Banishev, A.A.; Castillo-Garza, R.; Klimchitskaya, G.L.; Mostepanenko, V.M.; Mohideen, U. Gradient of the Casimir force between Au surfaces of a sphere and a plate measured using an atomic force microscope in a frequency-shift technique. Phys. Rev. B 2012, 85, 165443. [CrossRef]

38. Xu, J.; Klimchitskaya, G.L.; Mostepanenko, V.M.; Mohideen, U. Reducing detrimental electrostatic effects in Casimir-force measurements and Casimir-force-based microdevices. Phys. Rev. A 2018, 97, 032501. [CrossRef]

39. Blom, M. Seismic Attenuation for Advanced Virgo. Ph.D. Thesis, Vrije Universiteit Amsterdam, Amsterdam, The Netherlands, 2015.

40. Antezza, M.; Pitaevskii, L.P.; Stringari, S. Effect of the Casimir-Polder force on the collective oscillations of a trapped Bose-Einstein condensate. Phys. Rev. A 2004, 70, 053619. [CrossRef]

41. Speake, C.C.; Trenkel, C. Forces between Conducting Surfaces Due to Spatial Variations of Surface Potential. Phys. Rev. Lett. 2003, 90, 160403. [CrossRef] [PubMed]

42. Behunin, R.O.; Intravaia, F.; Dalvit, D.A.R.; Neto, P.A.M.; Reynaud, S. Modeling electrostatic patch effects in Casimir force measurements. Phys. Rev. A 2012, 85, 012504. [CrossRef]

43. Bressi, G.; Carugno, G.; Onofrio, R.; Ruoso, G. Measurement of the Casimir Force between Parallel Metallic Surfaces. Phys. Rev. Lett. 2002, 88, 041804. [CrossRef] [PubMed]

44. Antonini, P.; Bimonte, G.; Bressi, G.; Carugno, G.; Galeazzi, G.; Messineo, G.; Ruoso, G. An experimental apparatus for measuring the Casimir effect at large distunces. J. Phys. Conf. Ser. 2006, 161, 012006. [CrossRef]

45. Decca, R.S.; López, D.; Fischbach, E.; Klimchitskaya, G.L.; Krause, D.E.; Mostepanenko, V.M. Precise comparison of theory and new experiment for the Casimir force leads to stronger constraints on thermal quantum effects and long-range interactions. Ann. Phys. 2005, 318, 37-80. [CrossRef]

46. Decca, R.S.; López, D.; Chan, H.B.; Fischbach, E.; Krause, D.E.; Jamell, C.R. Constraining New Forces in the Casimir Regime Using the Isoelectronic Technique. Phys. Rev. Lett. 2005, 94, 240401. [CrossRef]

47. Chen, Y.J.; Tham, W.K.; Krause, D.E.; López, D.; Fischbach, E.; Decca, R.S. Stronger Limits on Hypothetical Yukawa Interactions in the 30-8000 Nm Range. Phys. Rev. Lett. 2016, 116, 221102. [CrossRef] [PubMed]

48. Wang, J.; Guan, S.; Chen, K.; Wu, W.; Tian, Z.; Luo, P.; Jin, A.; Yang, S.; Shao, C.; Luo, J. Test of non-Newtonian gravitational forces at micrometer range with two-dimensional force mapping. Phys. Rev. D 2016, 94, 122005. [CrossRef]

49. Masuda, M.; Sasaki, M. Limits on Nonstandard Forces in the Submicrometer Range. Phys. Rev. Lett. 2009, 102, 171101. [CrossRef]

50. Chiaverini, J.; Smullin, S.J.; Geraci, A.A.; Weld, D.M.; Kapitulnik, A. New Experimental Constraints on Non-Newtonian Forces below $100 \mu \backslash$. Phys. Rev. Lett. 2003, 90, 151101. [CrossRef]

51. Smullin, S.J.; Geraci, A.A.; Weld, D.M.; Chiaverini, J.; Holmes, S.; Kapitulnik, A. Constraints on Yukawa-type deviations from Newtonian gravity at 20 microns. Phys. Rev. D 2005, 72, 122001. [CrossRef]

52. Geraci, A.A.; Smullin, S.J.; Weld, D.M.; Chiaverini, J.; Kapitulnik, A. Improved constraints on non-Newtonian forces at 10 microns. Phys. Rev. D 2008, 78, 022002. [CrossRef]

53. Adelberger, E.G.; Fischbach, E.; Krause, D.E.; Newman, R.D. Constraining the couplings of massive pseudoscalars using gravity and optical experiments. Phys. Rev. D 2003, 68, 062002. [CrossRef]

54. Kim, J.E. Light pseudoscalars, particle physics and cosmology. Phys. Rep. 1987, 150, 1-177. [CrossRef]

55. Ferrer, F.; Nowakowski, M. Higgs- and Goldstone-boson-mediated long range forces. Phys. Rev. D 1999, 59, 075009. [CrossRef] 
56. Kapner, D.J.; Cook, T.S.; Adelberger, E.G.; Gundlach, J.H.; Heckel, B.R.; Hoyle, C.D.; Swanson, H.E. Tests of the Gravitational Inverse-Square Law below the Dark-Energy Length Scale. Phys. Rev. Lett. 2007, 98, 021101. [CrossRef]

57. Adelberger, E.G.; Heckel, B.R.; Hoedl, S.; Hoyle, C.D.; Kapner, D.J.; Upadhye, A. Particle-Physics Implications of a Recent Test of the Gravitational Inverse-Square Law. Phys. Rev. Lett. 2007, 98, 131104. [CrossRef]

58. Decca, R.S.; López, D.; Fischbach, E.; Klimchitskaya, G.L.; Krause, D.E.; Mostepanenko, V.M. Tests of new physics from precise measurements of the Casimir pressure between two gold-coated plates. Phys. Rev. D 2007, 75, 077101. [CrossRef]

59. Decca, R.S.; López, D.; Fischbach, E.; Klimchitskaya, G.L.; Krause, D.E.; Mostepanenko, V.M. Novel constraints on light elementary particles and extra-dimensional physics from the Casimir effect. Eur. Phys. J. C 2007, 51, 963. [CrossRef]

60. Chiu, H.C.; Klimchitskaya, G.L.; Marachevsky, V.N.; Mostepanenko, V.M.; Mohideen, U. Demonstration of the asymmetric lateral Casimir force between corrugated surfaces in the nonadditive regime. Phys. Rev. B 2009, 80, 121402. [CrossRef]

61. Chiu, H.C.; Klimchitskaya, G.L.; Marachevsky, V.N.; Mostepanenko, V.M.; Mohideen, U. Lateral Casimir force between sinusoidally corrugated surfaces: Asymmetric profiles, deviations from the proximity force approximation, and comparison with exact theory. Phys. Rev. B 2010,81, 115417. [CrossRef]

62. Wilson, C.M.; Johansson, G.; Pourkabirian, A.; Simoen, M.; Johansson, J.R.; Duty, T.; Nori, F.; Delsing, P. Observation of the dynamical Casimir effect in a superconducting circuit. Nature 2011, 479, 376-379 [CrossRef] [PubMed]

63. Felicetti, S.; Sanz, M.; Lamata, L.; Romero, G.; Johansson, G.; Delsing, P.; Solano, E. Dynamical Casimir Effect Entagles Artificial Atoms, Phys. Rev. Lett. 2014, 113, 093602. [CrossRef]

(C) 2019 by the authors. Licensee MDPI, Basel, Switzerland. This article is an open access article distributed under the terms and conditions of the Creative Commons Attribution (CC BY) license (http:/ / creativecommons.org/licenses/by/4.0/). 